\section{Device-guided breathing as a possible tool to improve the outcome of exposure therapy}

\author{
Maartje Cathelijne de Jong,' \\ Cornelis Hendrikus Boersma ${ }^{2}$
}

'Department Physics of Man, Helmholtz Institute/Utrecht University, Utrecht, The Netherlands

2Psychologiepraktijk Helmond, Helmond, The Netherlands

\begin{abstract}
Exposure therapy is a widely used treatment for patients with post-traumatic stress disorder. It involves reduction of fear through progressive exposure to frightening stimuli in a therapeutic environment. Here we propose a new method designed to improve the effectiveness of exposure therapy. We hypothesized that device-guided breathing during exposure therapy can increase the capability of the patient to undergo effective exposure. The successful application of the method is described for a single patient. Using a device to slow and regularize breathing, the patient was calmed and experienced a greater sense of control and a profound effect of the exposure. The use of the breathing-guiding device is believed to reduce arousal level and excitability of sympathetic "fight-flight" behaviors. The present study suggests that device-guided breathing integrated with exposure therapy may provide a practically feasible and potentially promising non-pharmacological treatment after trauma.
\end{abstract}

\section{Introduction}

Traumatic experiences like violent robbery, traffic accidents, sexual assault, or war trauma can have a dramatic effect on mental and physical health. The majority of people encounter some trauma over a lifetime and a substantial portion of them develops post-traumatic stress disorder (PTSD). The estimated lifetime prevalence of PTSD among adult Americans is about 8\%. ${ }^{1}$ Trauma survivors suffer various complaints including nightmares, flashbacks, hypervigilance, poor concentration, and sleep disturbance. $^{2}$ In exposure-based therapy, the traumatic event is relived in a controlled, therapeutic environment, by means of vivid imagination or by confrontation with objects related to the trauma. Video footage and virtual-reality environments may also be used. Effective exposure can decrease inadequate feelings of being "unsafe" and increase control over bodily reactions related to fear. ${ }^{3-5}$

Problematically, many patients experience anxiety and loss of control during therapeutic exposure sessions. This may contribute to delays in care and non-adherence to treatment and may even be harmful. ${ }^{4-7}$ Breathing exercises are used often in an attempt to calm patients that undergo exposure therapy, but these exercises require strong attention to one's own breathing pattern and are not carried out easily. Patients that fail to perform breathing exercises correctly can experience oxygen deprivation, dyspnea, and hyperventilation. ${ }^{8}$ These bodily reactions to incorrect breathing have a dramatic effect on the outcome of exposure therapy, as they increase fear instead of providing a sense of being safe.

Here we propose a new method to enable safe and effortless breathing training and improve the effectiveness of exposure therapy: the use of a device that guides the patient to slow and regular breathing. In contrast to traditional unaided breathing training, deviceguided breathing gives therapists the opportunity to monitor the patient's breathing pattern based on quantifiable measures. Moreover, the patient is guided to a therapeutic breathing pattern by means of personalized instructions. The musical instructions produced by the breathing-guiding device are based on the online analysis of the current breathing pattern (Figure 1). In this way the breathing pattern is modified at the patient's own pace, and ongoing changes in the breathing pattern are taken into account. Previous studies using deviceguided breathing for other indications $s^{9,10}$ have validated it as safe to use and effective in achieving slow and regular breathing.

Exposure therapy can be seen as a form of learning. Rather than being "erased", the association of the exposed object with fear is "overwritten" with a new "I am safe" association. The new association serves as an alternative to the original association with fear. ${ }^{11}$ However, this learning process takes time and requires that the patient remains calm and that the exposure is intense and repetitive. We hypothesized that device-guided breathing during exposure therapy can increase the capability of the patient to undergo effective exposure in a short time (Figure 2). A possible mechanism underlying this hypothesis may be reduced arousal level and reduced excitability of sympathetic "fight-flight" behaviors with slow and regular breathing. ${ }^{12}$ Preliminary observations of several $(>10)$ patients with PTSD suggested that this new approach has significant clinical potential as a treatment for trauma survivors without the use of medication. The present study documents its application on a single patient and proposes a model for its mechanism of functioning.
Correspondence: Maartje Cathelijne de Jong, Department Physics of Man, Helmholtz Institute/Utrecht University, Kruyt building room W205, Padualaan 8, 3584 CH Utrecht, The Netherlands. E-mail: m.c.dejong@uu.nl

Key words: exposure therapy, respiration, posttraumatic stress disorder, sympathetic arousal.

Contributions: MCdJ contributed to interpretation of data, drafting the article, and revising it critically for important intellectual content; CHB contributed to conception and design, and analysis and interpretation of data. Both authors approved the final version to be published.

Conflict of interest: the authors report no conflicts of interest.

Received for publication: 28 July 2009

Revision received: 16 April 2010.

Accepted for publication: 19 April 2010.

This work is licensed under a Creative Commons Attribution 3.0 License (by-nc 3.0).

(C) Copyright M.C.de Jong, C.H. Boersma, 2010

Licensee PAGEPress, Italy

Mental Illness 2010; 2:e6

doi:10.4081/mi.2010.e6

\section{Materials and Methods}

\section{Patient}

This case report describes the treatment of a 17-year-old Dutch male victim of random street violence, diagnosed with PTSD by a trained clinical psychologist using DSM-IV criteria. ${ }^{13}$ The patient reported nightmares, flashbacks, loss of concentration, and generalized fear of the outside world. His parents reported that he was hypervigilant, socially withdrawn, and not performing well at school. The patient had no comorbidities and no neurological or psychiatric history (no diagnosis on DSM-IV axis II, axis III, and axis IV, initial GAF 51-60). He was not using any medication.

\section{Breathing-guiding device}

The portable device (RESPeRATE ${ }^{\circledR}$, InterCure Ltd, Lod, Israel) used in this study analyzes breathing patterns by means of a belttype breathing sensor and creates, in real time, a two-tone inhale-exhale melody (Figure 1). The patient synchronizes breathing with these tones. It is a commercially available device indicated for treating stress and hypertension. It has already been found to be an effective treatment for hypertension ${ }^{9,12}$ and congestive heart failure. ${ }^{10}$ 


\section{Treatment}

In addition to the documented case, the treatment protocol described below was applied to several undocumented cases of PTSD ( $>10$ patients) and phobic disorder ( $<5$ patients).

Intake sessions: exchange of information

In one or two interviews of the patient by a therapist, details on the trauma/phobia and the symptoms of the patient were identified. Treatment procedures and rationale were explained and the patient (and/or caregivers) gave informed consent for participation in this study.

\section{Treatment sessions}

During 45- or 90-minute weekly individual treatment sessions, the patient was connected to the breathing-guiding device and instructed to effortlessly synchronize breathing with a twotone inhale-exhale melody produced by the device (Figure 1). In case the patient was uncomfortable with the indicated breathing pattern, the instruction was to breathe at the slowest comfortable pace. Holding the breath or putting effort into the breathing exercise was discouraged explicitly. Each session started with the device-guided breathing exercise. Exposure therapy was not started until the patient was comfortable with using the device and breathing frequency was below 8 breaths/minute. The exposure therapy either consisted of watching video-material related to the trauma/phobia or consisted of listening to and vividly imagining the events in a verbal story related to the trauma/phobia (told by a therapist). The patient was given full control over starting and stopping the exposure. Instructions were to (re)start the exposure only when breathing frequency was low ( $<8$ breaths/minute) and to stop the exposure as soon as breathing frequency increased (to $>8.5$ breaths/minute) or if feeling anxious or tense. When exposure was stopped the patient was instructed to use the device-guided breathing exercise to return to a breathing rate of less than 8 breaths/minute. A therapist observed the patient and his/her breathing frequency (shown on the display of the device) as unobtrusively as possible during the entire session. There was no homework-assignment between treatment sessions and the breathingguiding device was not used for practice at home. Treatment was stopped if, by the end of a session, the patient was able to undergo exposure continuously for 10 minutes while remaining at a low breathing frequency ( $<8$ breaths/ minute).

\section{Follow-up sessions}

The follow-up check-ups consisted of a telephone interview of the patient by a therapist. The symptoms of the patient as well as indications to provide additional treatment were carefully assessed.

\section{Results}

The patient was treated according to the treatment protocol described in the methods section. After two treatment sessions combining exposure therapy and device-guided breathing, he became symptom-free up to the last follow-up at two years after treatment. The patient found no difficulty in using the breathing-guiding device.

\section{First treatment session}

This 90-minute session was held approximately one month after the traumatic event. The patient was connected to the breathingguiding device (Figure 1) for approximately 75 minutes. In his hand the patient held the remote control of a DVD-player that contained video-material related to the trauma. After seven minutes of breathing exercise, the breathing frequency was 7 breaths/minute. The patient then started the video-clip and stopped it after approximately 15 seconds. He took several minutes to return to slow and regular breathing (with the use of the breathing-guiding device) before he restarted the video-clip. Subsequently, he intermittently watched the video-clip for increasing periods of time. Each time he started the video-clip his breathing frequency was around 8 breaths/ minute, and after he stopped it his breathing frequency was typically between 9 and 12 breaths/minute. By the end of the session he
A.

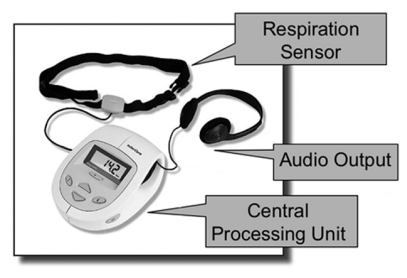

B.

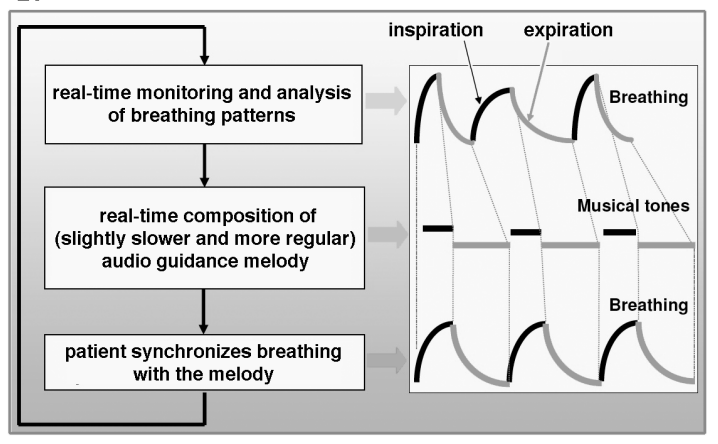

Figure 1. Operation of the breathing-guiding device. (A) The device consists of a computerized processing unit, a belt-type breathing sensor, and headphones for audio guidance. (B) The device provides personalized breathing instructions by means of a two-tone inhale-exhale melody that is based on the online analysis of breathing patterns. The patient synchronizes his/her breathing pattern with the musical tones (figures are obtained with permission from private communication with Benjamin Gavish).

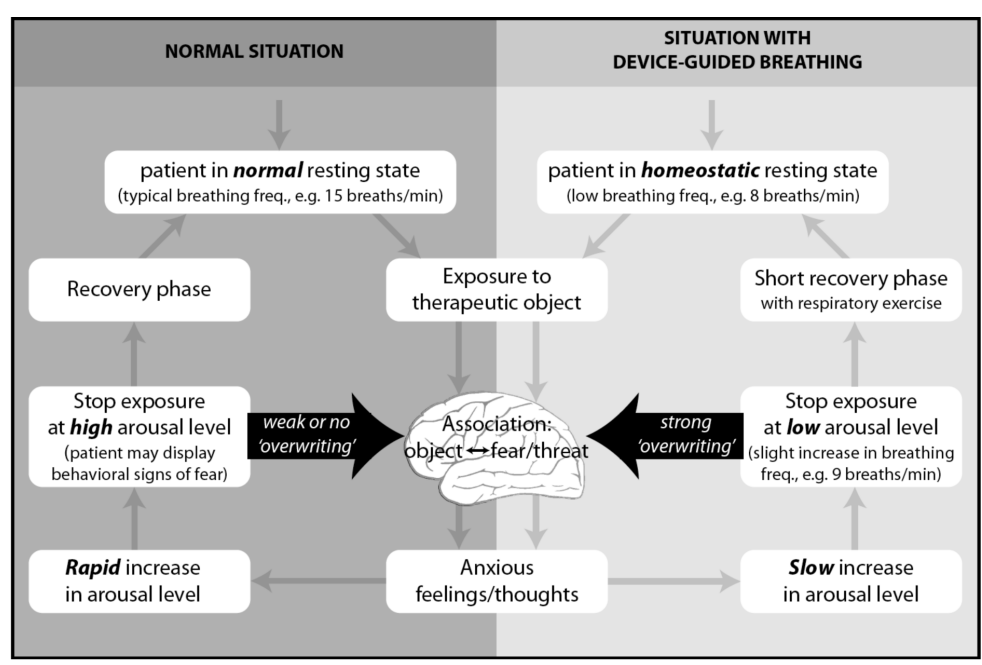

Figure 2. Proposed effect of device-guided breathing on the outcome of exposure therapy. The therapeutic object is an object, scene, or scenario related to the specific trauma or phobia of the patient. This object will trigger anxiety through a learned/remembered association with fear and threat. The purpose of exposure therapy is to "overwrite" this association with a new "I am safe" association. Effective learning of this new association can take place only if the patient is calm, i.e. has a low arousal level. A rapid increase in arousal level (sympathetic activity) during exposure therapy is prevented with deviceguided breathing by bringing the patient to a stable homeostatic resting state. 
was able to watch the video-clip for a few consecutive minutes. The total duration of the exposure during the entire session was approximately 11 minutes. In accordance with the treatment protocol (see methods section), a second treatment session was scheduled because the patient had not yet undergone 10 consecutive minutes of continuous exposure with slow breathing ( $<8$ breaths/minute).

\section{Second treatment session}

The patient reported that no nightmares had occurred since the previous session and that other symptoms had been greatly reduced. The procedure from the previous session was repeated. After 45 minutes it was decided to stop the treatment because the patient was able to watch the video clip for 10 consecutive minutes with only a slight increase in breathing frequency (from 8 to 9 breaths/minute). Although the images were violent, the patient reported to have a "sense of control" over his emotions/mood.

\section{Follow-up sessions}

During the follow-up sessions at one week, two months, and two years after treatment, the patient reported that he was symptom-free (GAF-score at two month's follow-up: 81-90).

\section{Discussion}

Exposure therapy is a very intensive type of treatment in which a patient is confronted with frightening stimuli or relives a traumatic event. The case study described here suggests that device-guided breathing increases the effectiveness of exposure therapy, thereby providing reduction of symptoms without the use of medication.

Normally, device-guided breathing is indicated for blood pressure reduction through extensive training at home. Here we describe a short-term therapeutic application: temporarily bringing the patient to a homeostatic resting state that is associated with rest and positive $\operatorname{mood}^{14,15}$ to increase the therapeutic effect of exposure-based treatment (Figure 2). This resting state is characterized by strong respiratory sinus arrhythmia; that is, an optimal tuning between breathing frequency, heart rate, and blood pressure that maximizes the body's energetic efficiency. ${ }^{15}$ Patients in this state show an increase in parasympathetic activity (particularly vagal tone) and a clear decrease in the excitability of sympathetic "fight-flight" reactions. ${ }^{14,16,17}$ Slow breathing may also inhibit sympathetic activity directly through the activation of pulmonary stretch receptors. ${ }^{12,18}$ Some forms of biofeedback might have comparable benefits provided that they influence the same homeostatic resting state (e.g. biofeedback to increase heart rate variability).

Device-guided breathing has several important advantages over breathing guidance by a therapist. With the breathing information that the device provides, it is much easier for the therapist to assess objectively whether the patient is performing the exercise correctly. Additionally, it is possible to describe the breathing exercise in a treatment protocol objectively and quantifiably. Device-guided breathing also requires less attention of the patient as well as the therapist, which is crucial when the breathing exercise is to be combined with exposure therapy. Furthermore, the personalized instructions calculated by the device reduce the chances of common negative effects of unaided breathing training, such as oxygen deprivation, dyspnea, and hyperventilation. ${ }^{8}$ The potential availability of device-guided breathing in the clinical practice is large, because the device is easy to use and affordable. We suggest this new approach is suited primarily for patients that show signs of autonomic arousal when talking or thinking about the trauma (such as tachycardia, rapid breathing, and flushing). An additional indication is a clearly definable trauma/phobia. Given the obvious limitations of uncontrolled case reports, future clinical and experimental research is needed to provide the necessary knowledge to develop this approach further as a standard of care. For example, it might be useful to monitor not only the breathing pattern but also the breathing volume. Additionally, the suitability of this treatment for phobic patients could be investigated.

The present work provides preliminary support for the use of device-guided breathing integrated with exposure therapy in the treatment of PTSD. As it is the first report of its kind, it may serve as a stepping-stone for further validation of this new approach. Given the frequent use of exposure therapy, this new approach may be of great clinical potential as a non-pharmacological treatment that is easy to implement in clinical practice.

\section{References}

1. Kessler RC, Sonnega A, Bromet E, et al. Posttraumatic stress disorder in the National Comorbidity Survey. Arch Gen Psychiatry 1995;52:1048-60.

2. Zohar J, Juven-Wetzler A, Myers V, et al. Post-traumatic stress disorder: facts and fiction. Curr Opin Psychiatry 2008;21:74-7.

3. Foa EB, Kozak MJ. Emotional processing of fear: Exposure to corrective information. Psychol Bull 1985;99:20-35.

4. Marks I, Lovell K, Noshirvani H. Treatment of posttraumatic stress disorder by exposure and/or cognitive restructuring: A controlled study. Arch Gen Psychiatry 1998;55: 317-25.

5. Rothbaum B0, Schwartz AC. Exposure therapy for posttraumatic stress disorder. Am J Psychother 2002;56:59-75.

6. Davis M, Ressler K, Rothbaum B0, et al. Effects of D-cycloserine on extinction: Translation from preclinical to clinical work. Biol Psychiatry 2006;60:369-75.

7. Mohlman J, Zinbarg RE. What kind of attention is necessary for fear reduction? An empirical test of the emotional processing model. Behav Ther 2000;31:113-33.

8. Conrad A, Müller A, Doberenz S, et al. Psychophysiological effects of breathing instructions for stress management. Appl Psychophysiol Biofeedback 2007;32:89-98.

9. Elliott WJ, Izzo JL. Device-guided breathing to lower blood pressure: case report and clinical overview. MedGenMed 2006; 8:23.

10. Parati G, Malfatto G, Boarin S, et al. Device-guided paced breathing in the home setting. On exercise capacity, pulmonary and ventricular function in patients with chronic heart failure: A pilot study. Circ Heart Fail 2008;1:178-83.

11. Milad MR, Rauch SL, Pitman RK, et al. Fear extinction in rats: Implications for human brain imaging and anxiety disorders. Biol Psychol 2006;73:61-71.

12. Parati G, Izzo J, Gavish B. Respiration and blood pressure. In: Hypertension Primer. 3rd edition. Izzo JL, Black HR, eds. Texas: American Heart Association, 2008, pp 36-138.

13. American Psychiatric Association. Diagnostic and statistical manual of mental disorders. 4th edition (DSM-IV). Washington, DC: 1994.

14. Friedman BH. An autonomic flexibilityneurovisceral integration model of anxiety and cardiac vagal tone. Biol Psychol 2007;74:185-99.

15. Hayano J, Yasuma F. Hypothesis: respiratory sinus arrhythmia is an intrinsic resting function of cardiopulmonary system. Cardiovasc Res 2003;58:1-9.

16. Asanoi H, Goso Y, Yamazaki T, et al. Slowing respiration effectively suppresses sympathetic nerve activity in patients with chronic heart failure. Circ J 2004;68:S184.

17. Vaschillo E, Vaschillo B, Lehrer P. Heartbeat synchronizes with respiratory rhythm only under specific circumstances. Chest 2004;126:1385-7.

18. Daly MdeB. Aspects of the integration of the respiratory and cardiovascular systems. In: Cardiovascular Regulation. Jordan D, Marshall JM, eds. London: Portland, 1994;15-35. 